\title{
Evaluation of Morphological Properties of Avenue Tree Species and Concentrations of Pollutants Under and Outside Their Canopies in Port Harcourt, Nigeria
}

\author{
Uzoma Darlington Chima (Corresponding author) \\ Department of Forestry and Wildlife Management, \\ Faculty of Agriculture, University of Port Harcourt, Nigeria \\ Tel: 234-803-812-1887. E-mail: uzoma.chima@uniport.edu.ng \\ Miriam Ugochi Opara \\ Department of Forestry and Wildlife Management, \\ Faculty of Agriculture, University of Port Harcourt, Nigeria \\ Tel: 234-703-525-2889. E-mail: lovedays4miriam@gmail.com
}

Received: August 3, 2019

doi:10.5296/jee.v10i2.15258
Accepted: August 28, 2019 Published: August 30, 2019

URL: https://doi.org/10.5296/jee.v10i2.15258

\begin{abstract}
We evaluated the morphological attributes of three major avenue tree species (Delonix regia, Gmelina arborea and Terminalia mantaly) and the concentrations of pollutants under and outside their canopies at four locations reflecting different levels of anthropogenic activities that contribute to pollution, in Port Harcourt, Rivers State, Nigeria. The sites are: Shell Petroleum Development Company (SPDC) Agbada (II) Flow Station, Aluu; LNG Seaport (LNG), Trans-Amadi; Isaac Boro Park (IBP), Mile 1; and Rivers State University (RSU), Nkporlu. Three trees belonging to each of the three species were randomly selected at each site and measurements of their total height (THT), diameter at breast height (DBH), crown diameter (CD), and basal area (BA), were taken. Concentrations of carbon monoxide (CO), nitrogen dioxide $\left(\mathrm{NO}_{2}\right)$, ammonia $\left(\mathrm{NH}_{3}\right)$, methane $\left(\mathrm{CH}_{4}\right)$, sulphur dioxide $\left(\mathrm{SO}_{2}\right)$, and particulate matter (PM) under and $50 \mathrm{~m}$ outside their canopies, were also measured. One-way Analysis of Variance (ANOVA) was used to test for significant difference $(p \leq 0.05)$ in tree attributes and the concentrations of pollutants under canopies of each tree species among sites, while t-test was used to test for significant difference $(p \leq 0.05)$ in the concentrations of the pollutants under and $50 \mathrm{~m}$ outside tree canopies at each site. THT, DBH, CD and BA of the three tree species varied significantly at the four sites with highest and lowest mean values
\end{abstract}


recorded for RSU and $\mathrm{LNG}$, respectively, except for D. regia where $\mathrm{DBH}$ and BA were highest and lowest at IBP and LNG, respectively. The concentration of pollutants under tree canopies at different sites did not vary significantly $(p>0.05)$ in most cases (except PM) despite the significant differences in tree growth/morphological attributes. There was no significant difference between the concentrations of pollutants under and $50 \mathrm{~m}$ outside tree canopies in all the sites. Higher concentrations of $\mathrm{CO}$ and PM were recorded under trees at LNG Seaport while $\mathrm{NO}_{2}, \mathrm{SO}_{2}$ and $\mathrm{CH}_{4}$ were higher under trees at SPDC, indicating that the level of pollutants at each site reflected the nature of the activities associated with the sites and not differences in tree morphological attributes. However, concentrations of the pollutants, except $\mathrm{CH}_{4}$, were below recommended exposure limits under and outside tree canopies, at all the locations.

Keywords: Air pollution, Avenue trees, Tree morphology, Port Harcourt City

\section{Introduction}

Avenue trees and urban forests are now being recognized as vital components of the urban landscape. Avenue trees are known to have both direct and indirect effects on street users (Heimlich et al., 2008); as their shade keeps pedestrians, parked cars, and surrounding buildings cool during sunny periods (Scott et al., 1999; Akbari, 2002). In an era of climate change and the associated global warming, trees in urban areas also provide means of adaptation to the adverse effects of torrential rains and damaging winds (Chima \& Ofodile, 2015). They equally help in beautifying the urban landscape (Roy \& Singh, 2014).

However, environmental pollution is becoming more threatening in many of our major cities as a result of rapid industrialization and urbanization. The air in some of our cities is being continuously contaminated through congested traffic, domestic fuel combustion, stone quarries, coalmines and various agricultural activities from the adjoining areas (Kumar et al., 2012). It is a recognized fact that air pollution poses huge threats to human health, and can cause airborne infections that result to common cold, bronchitis, asthma, hay fever, lung and respiratory diseases, heart attacks and premature deaths (Kumar et al., 2012).

In an environment like that of the Port Harcourt City, with a very high level of pollution, due to the presence and activities of oil producing companies, in addition to high fuel combustion resulting from congested traffic and other anthropogenic activities, avenue trees may also reduce the level of pollutants by absorbing and immobilizing them in their biomass. Avenue trees especially in urban cities also play very important roles in climate change adaptation. Adaptation to climate change has been defined as an adjustment in ecological structures, social or economic systems in response to actual or expected climatic stimuli and their effects or impacts (IPCC, 2001). Hence, adaptation is considered a plausible option in reducing vulnerability and the negative effects associated with climate change (Jones, 2010). They offer ecosystem services that enhance the adaptation of rural people to climate change and also the adaptation of wider sectors of the economy and society; therefore, they are recognized as key components of ecosystem-based adaptation (IUCN, 2009). Although, Ndubueze-Ogaraku and Emeh (2019) acknowledged the growing awareness on issues of climate change, they observed that the level of awareness amongst the very vulnerable groups, including women and children, is very low especially among the rural dwellers. 


\section{Macrothink

In urban areas on the other hand, the advocacy for tree planting for climate change mitigation and adaptation in recent times is quite encouraging. However, there has been no attempt to ascertain the safety or otherwise, in relaxing under canopies of avenue trees especially in urban and industrialized cities like Port Harcourt, or to ascertain if tree morphological properties of avenue trees influence their abilities to trap and keep pollutants under their canopies. This study was conceived and conducted to bridge this gap in knowledge.

\section{Materials and Methods}

\subsection{Study Area}

This study was carried out at four locations in Port Harcourt, Rivers State. Port Harcourt has a tropical climate. Rainfall is significant in most parts of the year. An average annual temperature of $27.2{ }^{\circ} \mathrm{C}$ for Port Harcourt has been reported by (Efe \& Weli, 2015). The natural vegetation in Port Harcourt has been cleared immensely due to urbanization though avenue and amenity trees are planted at different places.

\subsection{Selection of Study Sites}

Four sites reflecting different anthropogenic activities that contribute to pollution, in different parts Port Harcourt, were used for the study, following a reconnaissance survey that confirmed that the three avenue tree species -Delonix regia, Gmelina arborea and Terminalia mantaly, considered were present in each of them. The sites are: Shell Petroleum Development Company (SPDC) Agbada (II) Flow Station, Aluu; LNG Seaport, Trans-Amadi; Isaac Boro Park, Mile 1; and Rivers State University, Nkporlu. The Shell Petroleum Development Company (SPDC) Agbada (II) Flow Station $\left(7.01^{\circ} \mathrm{E}\right.$ and $4.93^{\circ} \mathrm{N}$ ), is an oil flow station which produces 25,000 barrels per day. It is a complement of Agbada (I) flow station located along Aluu road. Gas flaring is a major phenomenon in this site. The LNG Seaport belongs to the LNG Ship Manning Limited (NSML), a subsidiary of the Nigeria Liquefied Natural Gas (NLNG). It is located at $7.02^{\circ} \mathrm{E}$ and $4.79^{\circ} \mathrm{N}$ along eastern by-pass in Trans-Amadi, Port Harcourt, with their headquarters in Bonny Island, Rivers State. The presence of the Sea Port attracts a beehive of activities to the area and Trans-Amadi is generally known for hosting many oil services companies. Isaac Boro Park $\left(7.01^{\circ} \mathrm{E}\right.$ and $4.78^{\circ} \mathrm{N}$ ) is a public park and an outdoor recreation area situated opposite Mile 1 Bridge Motor Park, Port Harcourt. The Rivers State University (RSU) is a state owned university located at $6.98^{\circ} \mathrm{E}$ and $4.80^{\circ} \mathrm{N}$ in Nkporlu, Mile 3, Port Harcourt. Figure 1 is the map of Port Harcourt showing the study sites. 


\section{Macrothink}

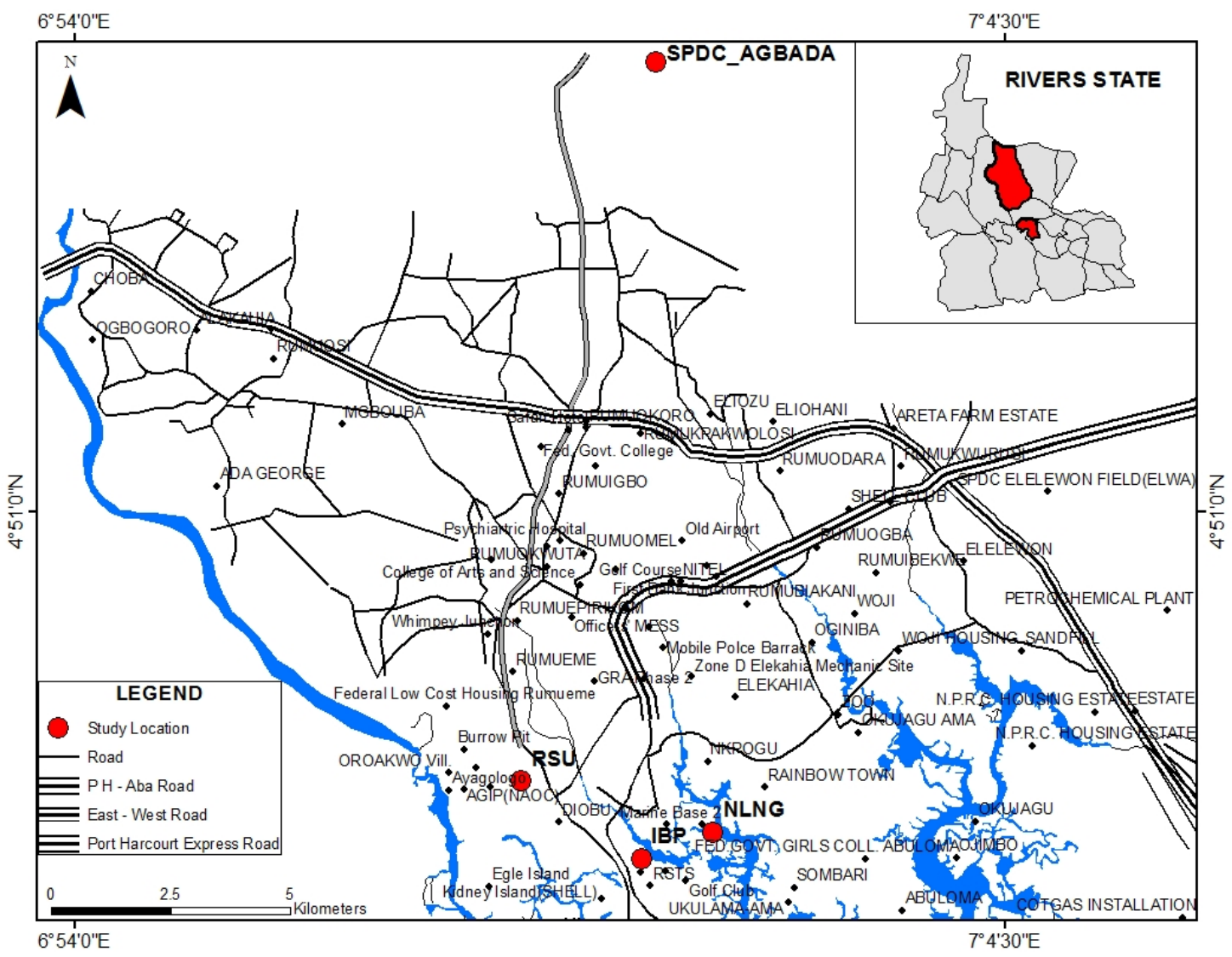

Figure 1. Map of Port Harcourt showing the study sites (Inset: Map of Rivers State showing the Study area)

\subsection{Data Collection}

\subsubsection{Selection of Sample Trees}

Three individual trees belonging to each of the three avenue tree species -Delonix regia, Gmelina arborea and Terminalia mantaly were randomly selected at each site for the measurement of some tree attributes, and the evaluation of pollutants under and outside their canopies.

\subsubsection{Measurement of Morphological Attributes of Trees}

Tree growth attributes that were evaluated are Diameter at Breast Height (DBH), Total Height (THT) and Crown Diameter (CD). Total height of each tree was measured using Haga altimeter. The diameter at breast height of each tree was estimated by first measuring the girth of the tree at $1.3 \mathrm{~m}$ from the base using a measuring tape. The girth was converted to diameter following White and Edwards (2000) with the formula: $\mathrm{DBH}=\mathrm{C} / \pi$ (where $\mathrm{C}=$ circumference or girth and $\pi=3.142$ ). The crown diameter was derived by taking the measurement of the distance between the tips of the crown from north to south and from east to west directions, using a measuring tape, and taking the average of the measurements in 
both directions (Chima et al., 2016). The Basal Area (BA) for individual trees was computed by first dividing the $\mathrm{DBH}$ of the tree by 2 to get the radius, squaring the radius and multiplying by $\pi$ to get the basal area (White \& Edwards, 2000).

\subsubsection{Measurement of Pollutants}

The ambient air under the canopies of the trees and $50 \mathrm{~m}$ outside the tree canopies was evaluated at each site for pollutants such as Carbon monoxide (CO) Sulphur dioxide $\left(\mathrm{SO}_{2}\right)$, Nitrogen dioxide $\left(\mathrm{NO}_{2}\right)$, Methane $\left(\mathrm{CH}_{4}\right)$, Ammonia $\left(\mathrm{NH}_{3}\right)$ and particulate matter $(\mathrm{PM})$. Carbon monoxide, sulphur dioxide, nitrogen dioxide, ammonia, and methane were measured using Industrial Scientific (IBRID) M X6 equipment, while particulate matter was measured using CEM Particle Counter (Model DT9880).

\subsection{Data Analysis}

\subsubsection{Analysis of Variance (ANOVA)}

One-way analysis of variance was used to test for significant difference $(p \leq 0.05)$ in the concentrations of pollutants under the canopy of each tree species and the measured tree morphological attributes, separately, among the sites. ANOVA was performed using the Statistical Package for Social Sciences (SPSS).

\subsubsection{T-test}

T-test was used to test for significant difference $(p \leq 0.05)$ in the concentrations of pollutants under and outside the canopy of each tree species in each site. T-test was performed using the Statistical Package for Social Sciences (SPSS).

\section{Results}

\subsection{Tree Growth Attributes at the various Study Sites}

The total heights of trees at the various sites are presented in Table 1 for the three species. The total heights of G. arborea and D. regia varied significantly among some sites with RSU and LNG having the highest and lowest mean heights, respectively. The total height of $T$. mantaly also varied significantly among some sites with IBP and LNG having the highest and lowest height, respectively.

Table 1. Total height of sample tree species at different study sites

\begin{tabular}{llll}
\hline Study Site & $\begin{array}{l}\text { Total height }(\mathrm{m}) \\
\text { Gmelina arborea }\end{array}$ & Terminalia mantaly & Delonix regia \\
\hline RSU & $21.80 \pm 2.32^{\mathrm{a}}$ & $18.57 \pm 1.93^{\mathrm{ad}}$ & $13.57 \pm 2.51^{\mathrm{a}}$ \\
IBP & $21.00 \pm 0.15^{\mathrm{ab}}$ & $22.57 \pm 2.23^{\mathrm{ab}}$ & $10.86 \pm 0.41^{\mathrm{abcd}}$ \\
LNG & $11.13 \pm 0.66^{\mathrm{c}}$ & $8.47 \pm 0.78^{\mathrm{c}}$ & $7.75 \pm 0.16^{\mathrm{c}}$ \\
SPDC & $14.73 \pm 2.31^{\mathrm{cd}}$ & $12.50 \pm 2.15^{\mathrm{cd}}$ & $12.37 \pm 0.75^{\mathrm{ad}}$ \\
\hline
\end{tabular}

Means on the same column with the same alphabet are not significantly different $(p>0.05)$.

RSU = Rivers State University, Nkporlu; IBP = Isaac Boro Park, Mile 1; LNG = LNG Seaport, Trans- Amadi; SPDC = Shell Flow Station Agbada II. 
The diameter at breast height ( $\mathrm{dbh}$ ) of the trees at the various sites is presented in Table 2 for the three species. The dbh of $G$. arborea varied significantly among some sites with the highest and the lowest mean values recorded at RSU and LNG, respectively. For T. mantaly and $D$. regia, dbh varied significantly among some sites but the highest and lowest mean values were recorded at IBP and LNG, respectively.

Table 2. Diameter at breast height of sample tree species at different study sites

\begin{tabular}{|c|c|c|c|}
\hline \multirow[t]{2}{*}{ Study Site } & \multicolumn{3}{|c|}{ Diameter at breast height (m) } \\
\hline & Gmelina arborea & Terminalia mantaly & Delonix regia \\
\hline RSU & $0.88 \pm 0.08^{\mathrm{ad}}$ & $0.59 \pm 0.04^{\mathrm{ad}}$ & $0.63 \pm 0.13^{\mathrm{a}}$ \\
\hline IBP & $0.82 \pm 0.05^{\mathrm{abd}}$ & $0.69 \pm 0.09^{\mathrm{ab}}$ & $0.70 \pm 0.04^{\mathrm{ab}}$ \\
\hline LNG & $0.47 \pm 0.06^{\mathrm{c}}$ & $0.38 \pm 0.04^{\mathrm{c}}$ & $0.27 \pm 0.02^{\mathrm{c}}$ \\
\hline SPDC & $0.67 \pm 0.13^{\mathrm{acd}}$ & $0.47 \pm 0.05^{\mathrm{cd}}$ & $0.39 \pm 0.02^{\mathrm{cd}}$ \\
\hline
\end{tabular}

Means on the same column with the same alphabet are not significantly different $(p>0.05)$. RSU = Rivers State University, Nkporlu; IBP = Isaac Boro Park, Mile 1; LNG = LNG Seaport, Trans- Amadi; SPDC = Shell Flow Station Agbada II.

The crown diameter of trees at the various sites is shown in Table 3. Tree crown diameter of the three species followed a similar trend with dbh. The crown diameter of $G$. arborea varied significantly among some sites with the highest and lowest mean values recorded at RSU and LNG, respectively. For $T$. mantaly and D. regia, crown diameter also varied significantly among some sites but the highest and lowest mean values for the two species were recorded at IBP and LNG, respectively.

Table 3. Crown diameter of sample tree species at different study sites

\begin{tabular}{llll}
\hline Study Site & $\begin{array}{l}\text { Crown diameter }(\mathrm{m}) \\
\text { Gmelina arborea }\end{array}$ & Terminalia mantaly & Delonix regia \\
\hline RSU & $18.49 \pm 1.42^{\mathrm{a}}$ & $17.37 \pm 1.65^{\mathrm{ad}}$ & $16.38 \pm 2.85^{\mathrm{ad}}$ \\
IBP & $16.47 \pm 1.31^{\mathrm{abd}}$ & $22.07 \pm 2.77^{\mathrm{ab}}$ & $20.68 \pm 0.82^{\text {abd }}$ \\
LNG & $10.08 \pm 1.30^{\mathrm{c}}$ & $7.13 \pm 0.42^{\mathrm{c}}$ & $6.55 \pm 0.22^{\mathrm{c}}$ \\
SPDC & $12.95 \pm 2.45^{\mathrm{bcd}}$ & $13.73 \pm 1.11^{\mathrm{d}}$ & $16.77 \pm 0.65^{\mathrm{d}}$ \\
\hline
\end{tabular}

Means on the same column with the same alphabet are not significantly different $(\mathrm{p}>0.05)$.

RSU $=$ Rivers State University, Nkporlu; IBP = Isaac Boro Park, Mile 1; LNG = LNG Seaport, Trans- Amadi; SPDC = Shell Flow Station Agbada II.

The basal area of sample trees at the various sites is shown in Table 4. The basal area of the tree species at the various sites followed a similar trend with dbh and crown diameter. The 
basal area of $G$. arborea varied significantly among some sites with the highest and lowest mean values recorded for RSU and LNG, respectively. For T. mantaly and D. regia, significant difference in basal area was also observed among some sites with the highest and lowest mean values recorded for IBP and LNG, respectively.

Table 4. Basal area of sample tree species at different study sites

\begin{tabular}{llll}
\hline Study Site & $\begin{array}{l}\text { Basal Area }\left(\mathrm{m}^{2}\right) \\
\text { Gmelina arborea }\end{array}$ & Terminalia mantaly & Delonix regia \\
\hline RSU & $0.62 \pm 0.11^{\mathrm{a}}$ & $0.28 \pm 0.04^{\mathrm{ab}}$ & $0.34 \pm 0.14^{\mathrm{ad}}$ \\
IBP & $0.53 \pm 0.06^{\mathrm{ab}}$ & $0.39 \pm 0.09^{\mathrm{b}}$ & $0.39 \pm 0.05^{\mathrm{ab}}$ \\
LNG & $0.18 \pm 0.04^{\mathrm{cd}}$ & $0.12 \pm 0.02^{\mathrm{ac}}$ & $0.06 \pm 0.01^{\mathrm{c}}$ \\
SPDC & $0.39 \pm 0.13^{\mathrm{abcd}}$ & $0.18 \pm 0.04^{\mathrm{ad}}$ & $0.12 \pm 0.01^{\mathrm{acd}}$ \\
\hline
\end{tabular}

Means on the same column with the same alphabet are not significantly different $(p>0.05)$.

RSU $=$ Rivers State University, Nkporlu; IBP $=$ Isaac Boro Park, Mile 1; LNG $=$ LNG Seaport, Trans- Amadi; SPDC = Shell Flow Station Agbada II.

\subsection{Concentrations of Pollutants under Tree Canopies at the various Study Sites}

The concentrations of Carbon monoxide (CO) under tree canopies at the various sites are shown in Table 5. Carbon monoxide concentrations under G. arborea and D. regia did not vary significantly between sites but the highest mean value was recorded at LNG for both species. However, CO under T. mantaly canopy varied significantly between LNG and each of the other sites with LNG having the highest mean value.

Table 5. Carbon monoxide (CO) concentrations under tree canopies at different sites

\begin{tabular}{llll}
\hline Study Site & CO $(\mathrm{ppm})$ & & \\
& Gmelina arborea & Terminalia mantaly & Delonix regia \\
\hline RSU & $1.00 \pm 0.00^{\mathrm{a}}$ & $1.00 \pm 0.00^{\mathrm{ad}}$ & $1.00 \pm 0.00^{\mathrm{a}}$ \\
IBP & $1.33 \pm 0.33^{\mathrm{a}}$ & $1.33 \pm 0.33^{\mathrm{abd}}$ & $1.00 \pm 0.00^{\mathrm{a}}$ \\
LNG & $2.00 \pm 0.58^{\mathrm{a}}$ & $6.67 \pm 0.88^{\mathrm{c}}$ & $2.37 \pm 1.17^{\mathrm{a}}$ \\
SPDC & $1.00 \pm 0.00^{\mathrm{a}}$ & $3.33 \pm 1.20^{\mathrm{d}}$ & $1.73 \pm 0.73^{\mathrm{a}}$ \\
\hline
\end{tabular}

Means on the same column with the same alphabet are not significantly different $(\mathrm{p}>0.05)$.

RSU = Rivers State University, Nkporlu; IBP = Isaac Boro Park, Mile 1; LNG = LNG Seaport, Trans- Amadi; SPDC = Shell Flow Station Agbada II.

Nitrogen dioxide $\left(\mathrm{NO}_{2}\right)$ concentrations (Table 6) under G. arborea were higher at SPDC and varied significantly between SPDC and other sites. However, there was no significant difference among sites in $\mathrm{NO}_{2}$ concentration under T. mantaly and D. regia. 
Table 6. Nitrogen dioxide $\left(\mathrm{NO}_{2}\right)$ concentrations under tree canopies at different sites

\begin{tabular}{llll}
\hline Study Site & $\begin{array}{l}\mathrm{NO}_{2}(\mathrm{ppm}) \\
\text { Gmelina arborea }\end{array}$ & Terminalia mantaly & Delonix regia \\
\hline $\mathrm{RSU}$ & $0.10 \pm 0.00^{\mathrm{a}}$ & $0.10 \pm 0.00^{\mathrm{a}}$ & $0.10 \pm 0.00^{\mathrm{a}}$ \\
$\mathrm{IBP}$ & $0.10 \pm 0.00^{\mathrm{ab}}$ & $0.10 \pm 0.00^{\mathrm{a}}$ & $0.10 \pm 0.00^{\mathrm{a}}$ \\
$\mathrm{LNG}$ & $0.10 \pm 0.00^{\mathrm{ac}}$ & $0.10 \pm 0.00^{\mathrm{a}}$ & $0.10 \pm 0.00^{\mathrm{a}}$ \\
SPDC & $0.70 \pm 0.30^{\mathrm{d}}$ & $0.50 \pm 0.25^{\mathrm{a}}$ & $0.07 \pm 0.03^{\mathrm{a}}$ \\
\hline
\end{tabular}

Means on the same column with the same alphabet are not significantly different $(p>0.05)$. RSU = Rivers State University, Nkporlu; IBP = Isaac Boro Park, Mile 1; LNG = LNG Seaport, Trans- Amadi; SPDC $=$ Shell Flow Station Agbada II.

The concentrations of Sulphur dioxide $\left(\mathrm{SO}_{2}\right)$ under tree canopies at the various sites are shown in Table 7. Higher concentrations of $\mathrm{SO}_{2}$ were recorded at SPDC under the three species, although there was no significant difference among sites.

Table 7. Sulphur dioxide $\left(\mathrm{SO}_{2}\right)$ concentrations under tree canopies at different sites

\begin{tabular}{llll}
\hline Study Site & $\begin{array}{l}\mathrm{SO}_{2}(\mathrm{ppm}) \\
\text { Gmelina arborea }\end{array}$ & Terminalia mantaly & Delonix regia \\
\hline $\mathrm{RSU}$ & $0.10 \pm 0.00^{\mathrm{a}}$ & $0.10 \pm 0.00^{\mathrm{b}}$ & $0.10 \pm 0.00^{\mathrm{c}}$ \\
$\mathrm{IBP}$ & $0.13 \pm 0.03^{\mathrm{a}}$ & $0.10 \pm 0.00^{\mathrm{b}}$ & $0.10 \pm 0.00^{\mathrm{c}}$ \\
$\mathrm{LNG}$ & $0.13 \pm 0.03^{\mathrm{a}}$ & $0.10 \pm 0.00^{\mathrm{b}}$ & $0.10 \pm 0.00^{\mathrm{c}}$ \\
$\mathrm{SPDC}$ & $0.67 \pm 0.03^{\mathrm{a}}$ & $0.20 \pm 0.00^{\mathrm{b}}$ & $0.13 \pm 0.03^{\mathrm{c}}$ \\
\hline
\end{tabular}

Means on the same column with the same alphabet are not significantly different $(p>0.05)$.

RSU = Rivers State University, Nkporlu; IBP = Isaac Boro Park, Mile 1; LNG = LNG Seaport, Trans- Amadi; SPDC = Shell Flow Station Agbada II.

The concentrations of methane (Table 8) did not vary significantly among sites under the three species. However, the highest concentration was recorded under T. mantaly at SPDC.

Table 8. Methane $\left(\mathrm{CH}_{4}\right)$ concentrations under tree canopies at different sites

\begin{tabular}{llll}
\hline Study Site & $\mathrm{CH}_{4}(\%)$ & & \\
& Gmelina arborea & Terminalia mantaly $^{(}$ & Delonix regia \\
\hline RSU & $1.00 \pm 0.00^{\mathrm{a}}$ & $1.00 \pm 0.00^{\mathrm{b}}$ & $1.00 \pm 0.00^{\mathrm{c}}$ \\
IBP & $1.00 \pm 0.00^{\mathrm{a}}$ & $1.00 \pm 0.00^{\mathrm{b}}$ & $1.00 \pm 0.00^{\mathrm{c}}$ \\
LNG & $1.00 \pm 0.00^{\mathrm{a}}$ & $1.33 \pm 0.33^{\mathrm{b}}$ & $1.00 \pm 0.00^{\mathrm{c}}$ \\
\hline
\end{tabular}




$\begin{array}{llll}\text { SPDC } & 1.00 \pm 0.00^{\mathrm{a}} & 1.67 \pm 0.33^{\mathrm{b}} & 1.00 \pm 0.00^{\mathrm{c}}\end{array}$

Means on the same column with the same alphabet are not significantly different $(p>0.05)$.

RSU = Rivers State University, Nkporlu; IBP = Isaac Boro Park, Mile 1; LNG = LNG Seaport, Trans- Amadi; SPDC = Shell Flow Station Agbada II.

The Concentrations of Ammonia (Table 9) did not vary significantly among sites under the three species. However, a higher concentration was recorded at LNG under G. arborea while the concentration was higher at SPDC under T. mantaly and D. regia.

Table 9. Ammonia $\left(\mathrm{NH}_{3}\right)$ concentrations under tree canopies at different sites

\begin{tabular}{llll}
\hline Study Site & $\mathrm{NH}_{3}(\mathrm{ppm})$ & & \\
& Gmelina arborea & Terminalia mantaly & Delonix regia \\
\hline RSU & $1.00 \pm 0.00^{\mathrm{a}}$ & $1.00 \pm 0.00^{\mathrm{b}}$ & $1.00 \pm 0.00^{\mathrm{c}}$ \\
$\mathrm{IBP}$ & $1.00 \pm 0.00^{\mathrm{a}}$ & $1.00 \pm 0.00^{\mathrm{b}}$ & $1.00 \pm 0.00^{\mathrm{c}}$ \\
LNG & $1.33 \pm 0.33^{\mathrm{a}}$ & $1.00 \pm 0.00^{\mathrm{b}}$ & $1.00 \pm 0.00^{\mathrm{c}}$ \\
SPDC & $1.00 \pm 0.00^{\mathrm{a}}$ & $1.33 \pm 0.33^{\mathrm{b}}$ & $1.33 \pm 0.33^{\mathrm{c}}$ \\
\hline
\end{tabular}

Means on the same column with the same alphabet are not significantly different $(p>0.05)$.

RSU = Rivers State University, Nkporlu; IBP = Isaac Boro Park, Mile 1; LNG = LNG Seaport, Trans- Amadi; SPDC = Shell Flow Station Agbada II.

Particulate matter under the sample trees at the various sites is shown in Table 10. Particulate matter under $G$. arborea and $T$. mantaly varied significantly among sites except for RSU/SPDC under G. arborea and RSU/SPDC and IBP/SPDC under T. mantaly, with the highest and lowest mean values recorded for LNG and RSU, respectively. Under D. regia, particulate matter varied significantly between LNG and each of the three other sites, with LNG having the highest mean value.

Table 10. Particulate matter (PM) concentrations under tree canopies at different sites

\begin{tabular}{llll}
\hline Study Site & PM $(\mathrm{p} / \mathrm{cf})$ & & \\
& Gmelina arborea & Terminalia mantaly & Delonix regia \\
\hline RSU & $11780 \pm 170.83^{\mathrm{a}}$ & $12073 \pm 72.72^{\mathrm{a}}$ & $12694 \pm 437.20^{\mathrm{ad}}$ \\
IBP & $13211 \pm 348.03^{\mathrm{b}}$ & $13248 \pm 148.45^{\mathrm{bd}}$ & $13259 \pm 138.86^{\mathrm{ab}}$ \\
LNG & $17084 \pm 41.19^{\mathrm{c}}$ & $17381 \pm 216.81^{\mathrm{c}}$ & $17556 \pm 294.74^{\mathrm{c}}$ \\
SPDC & $12320 \pm 99.17^{\text {ad }}$ & $12701 \pm 510.29^{\text {ad }}$ & $12272 \pm 78.00^{\mathrm{d}}$ \\
\hline
\end{tabular}

Means on the same column with the same alphabet are not significantly different $(p>0.05)$. RSU $=$ Rivers State University, Nkporlu; IBP $=$ Isaac Boro Park, Mile 1; LNG = LNG 
Seaport, Trans- Amadi; SPDC = Shell Flow Station Agbada II.

\subsection{Comparative Evaluation of Pollutants' Concentrations under and outside Tree Canopies}

A comparative evaluation of the pollutants' concentrations under and outside tree canopies is presented in Table 11 for RSU, Table 12 for IBP, Table 13 for LNG and Table 14 for SPDC. Results in all the sites show that there was no significant difference in the pollutants' concentrations under and outside tree canopies in all the sites.

Table 11. Concentrations of pollutants under and outside tree canopies at RSU

\begin{tabular}{|c|c|c|c|c|c|c|c|c|c|c|c|c|}
\hline \multirow[t]{2}{*}{ Tree species } & \multicolumn{2}{|c|}{$\mathrm{CO}(\mathrm{ppm})$} & \multicolumn{2}{|c|}{$\mathrm{NO}_{2}(\mathrm{ppm})$} & \multicolumn{2}{|c|}{$\mathrm{SO}_{2}(\mathrm{ppm})$} & \multicolumn{2}{|l|}{$\mathrm{CH}_{4}(\%)$} & \multicolumn{2}{|c|}{$\mathrm{NH}_{3}(\mathrm{ppm})$} & \multicolumn{2}{|l|}{$\operatorname{PM}(\mathrm{p} / \mathrm{cf})$} \\
\hline & UTC & OTC & UTC & OTC & UTC & OTC & UTC & OTC & UTC & OTC & UTC & OTC \\
\hline G. arborea & $1.0 \pm 0.0^{\mathrm{a}}$ & $1.0 \pm 0.0^{\mathrm{a}}$ & $0.1 \pm 0.0^{\mathrm{b}}$ & $0.10 \pm 0.0^{\mathrm{b}}$ & $0.1 \pm 0.0^{\mathrm{c}}$ & $0.1 \pm 0.0^{\mathrm{c}}$ & $0.1 \pm 0.0^{\mathrm{d}}$ & $0.1 \pm 0.0^{\mathrm{d}}$ & $0.1 \pm 0.0^{\mathrm{e}}$ & $0.1 \pm 0.0^{\mathrm{e}}$ & $11780 \pm 170.8^{\mathrm{f}}$ & $11867 \pm 194.3^{\mathrm{f}}$ \\
\hline T. mantaly & $1.0 \pm 0.0^{\mathrm{a}}$ & $0.7 \pm 0.0^{\mathrm{a}}$ & $0.1 \pm 0.0^{\mathrm{b}}$ & $0.10 \pm 0.0^{\mathrm{b}}$ & $0.1 \pm 0.0^{\mathrm{c}}$ & $0.1 \pm 0.0^{\mathrm{c}}$ & $0.1 \pm 0.0^{\mathrm{d}}$ & $0.1 \pm 0.0^{\mathrm{d}}$ & $0.1 \pm 0.0^{\mathrm{e}}$ & $1.3 \pm 0.3^{\mathrm{e}}$ & $12073 \pm 72.8^{\mathrm{f}}$ & $12033 \pm 44.1^{\mathrm{f}}$ \\
\hline D. regia & $1.0 \pm 0.0^{\mathrm{a}}$ & $1.0 \pm 0.0^{\mathrm{a}}$ & $0.1 \pm 0.0^{\mathrm{b}}$ & $0.10 \pm 0.0^{\mathrm{b}}$ & $0.1 \pm 0.0^{\mathrm{c}}$ & $0.1 \pm 0.0^{\mathrm{c}}$ & $0.1 \pm 0.0^{\mathrm{d}}$ & $0.1 \pm 0.0^{\mathrm{d}}$ & $0.1 \pm 0.0^{\mathrm{e}}$ & $1.3 \pm 0.3^{\mathrm{e}}$ & $12694 \pm 437.2^{\mathrm{f}}$ & $14438 \pm 1911.7^{\mathrm{f}}$ \\
\hline
\end{tabular}

Means on the same row for each pollutant are not significantly different $(p>0.05)$.

$\mathrm{UTC}=$ Under Tree Canopy; OTC $=50 \mathrm{~m}$ Outside Tree Canopy.

Table 12. Concentrations of pollutants under and outside tree canopies at IBP

\begin{tabular}{lllllllllllll}
\hline Tree species & $\mathrm{CO}(\mathrm{ppm})$ & \multicolumn{2}{c}{$\mathrm{NO}_{2}(\mathrm{ppm})$} & $\mathrm{SO}_{2}(\mathrm{ppm})$ & $\mathrm{CH}_{4}(\%)$ & & $\mathrm{NH}_{3}(\mathrm{ppm})$ & \multicolumn{2}{l}{$\mathrm{PM}(\mathrm{p} / \mathrm{cf})$} \\
& $\mathrm{UTC}$ & OTC & UTC & OTC & UTC & OTC & UTC & OTC & UTC & OTC & UTC & OTC \\
\hline G. arborea & $1.3 \pm 0.3^{\mathrm{a}}$ & $1.0 \pm 0.0^{\mathrm{a}}$ & $0.1 \pm 0.0^{\mathrm{b}}$ & $0.1 \pm 0.0^{\mathrm{b}}$ & $0.1 \pm 0.0^{\mathrm{c}}$ & $0.1 \pm 0.0^{\mathrm{c}}$ & $1.0 \pm 0.0^{\mathrm{d}}$ & $1.0 \pm 0.0^{\mathrm{d}}$ & $1.0 \pm 0.0^{\mathrm{e}}$ & $1.0 \pm 0.0^{\mathrm{e}}$ & $13211 \pm 348.0^{\mathrm{f}}$ & $13381 \pm 33.1^{\mathrm{f}}$ \\
T. mantaly & $1.3 \pm 0.3^{\mathrm{a}}$ & $2.0 \pm 1.0^{\mathrm{a}}$ & $0.1 \pm 0.0^{\mathrm{b}}$ & $0.1 \pm 0.0^{\mathrm{b}}$ & $0.1 \pm 0.0^{\mathrm{c}}$ & $0.1 \pm 0.0^{\mathrm{c}}$ & $1.0 \pm 0.0^{\mathrm{d}}$ & $1.0 \pm 0.0^{\mathrm{d}}$ & $1.0 \pm 0.0^{\mathrm{e}}$ & $1.0 \pm 0.0^{\mathrm{e}}$ & $13248 \pm 148.5^{\mathrm{f}}$ & $13752 \pm 370.9^{\mathrm{f}}$ \\
D. regia & $1.0 \pm 0.0^{\mathrm{a}}$ & $1.0 \pm 0.0^{\mathrm{a}}$ & $0.1 \pm 0.0^{\mathrm{b}}$ & $0.1 \pm 0.0^{\mathrm{b}}$ & $0.1 \pm 0.0^{\mathrm{c}}$ & $0.1 \pm 0.0^{\mathrm{c}}$ & $1.0 \pm 0.0^{\mathrm{d}}$ & $1.0 \pm 0.0^{\mathrm{d}}$ & $1.0 \pm 0.0^{\mathrm{e}}$ & $1.0 \pm 0.0^{\mathrm{e}}$ & $13259 \pm 138.6^{\mathrm{f}}$ & $13186 \pm 61.7^{\mathrm{f}}$ \\
\hline
\end{tabular}

Means on the same row for each pollutant are not significantly different $(\mathrm{p}>0.05)$.

$\mathrm{UTC}=$ Under Tree Canopy; OTC $=50 \mathrm{~m}$ Outside Tree Canopy.

Table 13. Concentrations of pollutants under and outside tree canopies at LNG

\begin{tabular}{|c|c|c|c|c|c|c|c|c|c|c|c|c|}
\hline \multirow[t]{2}{*}{ Tree species } & \multicolumn{2}{|c|}{$\mathrm{CO}(\mathrm{ppm})$} & \multicolumn{2}{|c|}{$\mathrm{NO}_{2}(\mathrm{ppm})$} & \multicolumn{2}{|c|}{$\mathrm{SO}_{2}(\mathrm{ppm})$} & \multicolumn{2}{|l|}{$\mathrm{CH}_{4}(\%)$} & \multicolumn{2}{|c|}{$\mathrm{NH}_{3}(\mathrm{ppm})$} & \multicolumn{2}{|l|}{$\mathrm{PM}(\mathrm{p} / \mathrm{cf})$} \\
\hline & UTC & OTC & UTC & отC & UTC & ОтС & UTC & OTC & UTC & отC & UTC & OTC \\
\hline G. arborea & $2.0 \pm 0.6^{\mathrm{a}}$ & $6.3 \pm 2.9^{\mathrm{a}}$ & $0.1 \pm 0.0^{\mathrm{b}}$ & $0.1 \pm 0.0^{\mathrm{b}}$ & $0.1 \pm 0.0^{\mathrm{c}}$ & $0.1 \pm 0.0^{\mathrm{c}}$ & $1.0 \pm 0.0^{\mathrm{d}}$ & $1.0 \pm 0.0^{\mathrm{d}}$ & $1.3 \pm 0.3^{\mathrm{e}}$ & $1.0 \pm 0.0^{\mathrm{e}}$ & $17084 \pm 41.2^{\mathrm{f}}$ & $17259 \pm 84.8^{\mathrm{f}}$ \\
\hline T. mantaly & $6.7 \pm 0.9^{\mathrm{a}}$ & $6.7 \pm 0.9^{\mathrm{a}}$ & $0.1 \pm 0.0^{\mathrm{b}}$ & $0.1 \pm 0.0^{\mathrm{b}}$ & $0.1 \pm 0.0^{\mathrm{c}}$ & $0.1 \pm 0.0^{\mathrm{c}}$ & $1.3 \pm 0.3^{\mathrm{d}}$ & $1.0 \pm 0.0^{\mathrm{d}}$ & $1.0 \pm 0.0^{\mathrm{e}}$ & $1.0 \pm 0.0^{\mathrm{e}}$ & $17381 \pm 216.8^{f}$ & $17385 \pm 233.4^{\mathrm{f}}$ \\
\hline D. regia & $2.4 \pm 1.2^{\mathrm{a}}$ & $5.3 \pm 0.9^{\mathrm{a}}$ & $0.1 \pm 0.0^{\mathrm{b}}$ & $0.1 \pm 0.0^{\mathrm{b}}$ & $0.1 \pm 0.0^{\mathrm{c}}$ & $0.1 \pm 0.0^{\mathrm{c}}$ & $1.0 \pm 0.0^{\mathrm{d}}$ & $1.0 \pm 0.0^{\mathrm{d}}$ & $1.0 \pm 0.0^{\mathrm{e}}$ & $1.3 \pm 0.3^{\mathrm{e}}$ & $17556 \pm 294.7^{f}$ & $17539 \pm 193.5^{f}$ \\
\hline
\end{tabular}

Means on the same row for each pollutant are not significantly different $(\mathrm{p}>0.05)$.

$\mathrm{UTC}=$ Under Tree Canopy; OTC $=50 \mathrm{~m}$ Outside Tree Canopy. 
Table 14. Concentrations of pollutants under and outside tree canopies at SPDC

\begin{tabular}{|c|c|c|c|c|c|c|c|c|c|c|c|c|}
\hline \multirow[t]{2}{*}{ Tree species } & \multicolumn{2}{|c|}{$\mathrm{CO}(\mathrm{ppm})$} & \multicolumn{2}{|c|}{$\mathrm{NO}_{2}(\mathrm{ppm})$} & \multicolumn{2}{|c|}{$\mathrm{SO}_{2}(\mathrm{ppm})$} & \multicolumn{2}{|l|}{$\mathrm{CH}_{4}(\%)$} & \multicolumn{2}{|c|}{$\mathrm{NH}_{3}(\mathrm{ppm})$} & \multicolumn{2}{|l|}{$\mathrm{PM}(\mathrm{p} / \mathrm{cf})$} \\
\hline & UTC & OTC & UTC & OTC & UTC & OTC & UTC & OTC & UTC & OTC & UTC & OTC \\
\hline G. arborea & $1.0 \pm 0.0^{\mathrm{a}}$ & $0.7 \pm 0.3^{\mathrm{a}}$ & $0.7 \pm 0.3^{\mathrm{b}}$ & $0.7 \pm 0.3^{b}$ & $0.2 \pm 0.0^{\mathrm{c}}$ & $0.2 \pm 0.0^{\mathrm{c}}$ & $1.0 \pm 0.0^{\mathrm{d}}$ & $1.0 \pm 0.0^{\mathrm{d}}$ & $1.0 \pm 0.0^{\mathrm{e}}$ & $1.0 \pm 0.0^{\mathrm{e}}$ & $12320 \pm 99.2^{\mathrm{f}}$ & $12288 \pm 92.4^{\mathrm{f}}$ \\
\hline T. mantaly & $3.3 \pm 1.2^{\mathrm{a}}$ & $3.0 \pm 1.7^{\mathrm{a}}$ & $0.5 \pm 0.3^{\mathrm{b}}$ & $0.1 \pm 0.0^{\mathrm{b}}$ & $0.2 \pm 0.0^{\mathrm{c}}$ & $0.2 \pm 0.1^{\mathrm{c}}$ & $1.7 \pm 0.3^{\mathrm{d}}$ & $1.0 \pm 0.0^{\mathrm{d}}$ & $1.3 \pm 0.3^{\mathrm{e}}$ & $1.0 \pm 0.0^{\mathrm{e}}$ & $12701 \pm 510.3^{\mathrm{f}}$ & $12567 \pm 185.0^{\mathrm{f}}$ \\
\hline D. regia & $1.7 \pm 0.7^{\mathrm{a}}$ & $1.0 \pm 0.0^{\mathrm{a}}$ & $0.1 \pm 0.0^{\mathrm{b}}$ & $0.1 \pm 0.0^{b}$ & $0.1 \pm 0.0^{\mathrm{c}}$ & $0.4 \pm 0.2^{\mathrm{c}}$ & $1.0 \pm 0.0^{\mathrm{d}}$ & $1.0 \pm 0.0^{\mathrm{d}}$ & $1.3 \pm 0.3^{\mathrm{e}}$ & $1.0 \pm 0.0^{\mathrm{e}}$ & $12272 \pm 78.0^{\mathrm{f}}$ & $12355 \pm 121.7^{\mathrm{f}}$ \\
\hline
\end{tabular}

Means on the same row for each pollutant are not significantly different $(p>0.05)$.

$\mathrm{UTC}=$ Under Tree Canopy; OTC $=50 \mathrm{~m}$ Outside Tree Canopy.

\section{Discussion}

Total height, diameter at breast height, crown diameter and basal area of the three avenue tree species varied significantly among the study sites in most cases. These variations may be attributed to differences in age of the trees at the different study sites and/or variations in nutrient contents of their soils. Heimlich et al. (2008) observed that tree growth and attributes vary for different species of trees and also for trees of the same species in different environments. Apart from the probable differences in age and soil quality, the significant differences observed in the growth attributes at the various study sites especially diameter at breast height and crown diameter may be due to the variations in spacing between individual trees at the different study sites and other growth determinants including genetic differences.

The cause of primary emissions of Carbon monoxide is incomplete combustion. Exhaust of motor vehicles accounts for $85-95 \%$ of fossil fuel sources (US EPA, 2007). The impairment of the circulatory system with respect to the transportation of oxygen $\left(\mathrm{O}_{2}\right)$, reduced alertness on tasks requiring vigilance and worsening cases of cardiovascular diseases can be caused by high concentrations of Carbon monoxide (Jimoda, 2012). It was observed at the time of study, that dredging activities and constructions were ongoing at LNG-Seaport site. These activities may have contributed to more emissions of Carbon monoxide leading to higher mean values. However, the concentrations of Carbon monoxide at the LNG-Seaport and the other sites were below the $35 \mathrm{ppm}$ recommended exposure limit over an eight-hour time-weighted average by National Institute for Occupational Safety and Health of the United States of America (NIOSH) (1992).

Nitrogen dioxide $\left(\mathrm{NO}_{2}\right)$ is an unpleasantly smelling, poisonous (under high concentrations), reddish-brown gas capable of forming secondary pollutants that can have environmental consequences such as acidification and nitrogen enrichment (SEPA, 2017). Anthropogenic sources such as mobile combustion sources including road, air and water traffic and stationary combustion sources like industrial combustion, are responsible for emissions of nitrogen oxides. Nitrogen dioxide $\left(\mathrm{NO}_{2}\right)$ concentrations under G. arborea were higher at SPDC Flow Station Site, and varied significantly between it and the other sites. Higher concentrations of $\mathrm{NO}_{2}$ under the three tree species as was observed in SPDC could be as a result of immense gas flaring at the site. Many health issues including respiratory symptoms, especially among asthmatic children, and respiratory-related emergency department visits and hospital 
admissions, particularly for children and older adults, have been linked to exposure to $\mathrm{NO}_{2}$ (US EPA, 2008), although our results show that concentrations at the various sites were still within the recommended exposure limit of 1 ppm, at all times, by NIOSH (1992).

Similarly, higher concentrations of $\mathrm{SO}_{2}$ were recorded at SPDC Flow Station site under the canopies of the three tree species. However, the concentrations were generally below the recommended exposure limit of $2 \mathrm{ppm}$ for a ten-hour work shift and $5 \mathrm{ppm}$ at a short time exposure of 15-minutes work period, according to NIOSH (1992). There is the need to check the rising concentration of $\mathrm{SO}_{2}$ especially at the SPDC Flow Station site since exposure to $\mathrm{SO}_{2}$ beyond the permissible limit is known to adversely affect human health.

Although the concentration of methane did not vary significantly under the canopies of each of the three avenue tree species among the various sites, the highest concentration was recorded under T. mantaly at SPDC Flow Station site, followed by LNG-Seaport site under the same tree species. It should be noted that the concentrations of methane under the canopies of the three tree species were above the permissible limit, at all the study sites. This calls for concern as the maximum recommended safe methane concentration for workers during an eight-hour period according to NIOSH (1992), is $1000 \mathrm{ppm}(0.1 \%)$. The highest concentration of methane at the SPDC Flow Station site is as a result of gas flaring. Methane is emitted to the atmosphere during the production, processing, storage, transmission, and distribution of natural gas and also, when animals' manure is stored or managed in lagoons or holding tanks, $\mathrm{CH}_{4}$ is produced (EPA, 2010). At an extremely high concentration in an enclosed space, methane can cause reduction in oxygen level which can eventually lead to suffocation. In addition, the global warming potential of methane ranks higher (twenty-one times) than that of carbon dioxide.

The Concentrations of Ammonia under the canopies of the three tree species did not vary significantly among sites and were far below the maximum recommended limit. NIOSH (1992) had pegged the recommended exposure limit for ammonia to be $25 \mathrm{ppm}$ at an eight-hour time weighted average and $35 \mathrm{ppm}$ at a short-term exposure limit. The largest source of $\mathrm{NH}_{3}$ emissions is agriculture, including animal husbandry and $\mathrm{NH}_{3}$-based fertilizer applications. This could explain why the concentrations of $\mathrm{NH}_{3}$ at the various sites were so low since none of the sites is used for agriculture and animal husbandry. Other sources of $\mathrm{NH}_{3}$ include industrial processes, vehicular emissions and volatilization from soils and oceans (Bahera et al., 2013).

The concentration of particulate matter at the various sites was very high with the highest and lowest mean values recorded for the LNG Seaport site and RSU site, respectively. Moreover, the concentration of particulate matter at each site reflected the nature of the activities going on at the time of study or that goes on at the sites. For instance, construction work was going on at LNG Seaport site at the period of study while RSU which had the lowest mean value had reduced activities as the students were on vacation and no construction work was ongoing. In the urban environment, reductions in air quality resulting from emissions of particulate matter, primarily from road traffic, are a serious health issue globally (Yang et al., 2005). High concentration of suspended particulate matter in the atmosphere within the Niger Delta Region of Nigeria had been reported by Ede and Edokpa (2015).

Generally, there was no significant difference in the concentrations of pollutants under and outside canopies of the trees at the various sites despite the observed significant differences in the measured tree growth/morphological attributes among the various sites. There was also no 
particular order or trend observed in the concentration of pollutants under and outside tree canopies. For instance, the concentrations of some pollutants were higher under tree canopies while some were higher outside tree canopies. Although, it is an established fact that trees can absorb gases and lower their concentrations in the environment, Benneth and Hill (2003) observed that trees can also contribute to air pollution by emitting various chemicals. Our observations showed that there is no direct or definite relationship between the evaluated tree growth/morphological attributes and the trend in the concentration of pollutants under tree canopies on one hand, and between under and outside tree canopies on the other hand.

\section{Conclusion}

The concentrations of the evaluated pollutants under tree canopies at the various locations were found to be below the permissible exposure limits except for methane $\left(\mathrm{CH}_{4}\right)$. However, the concentrations of the pollutants were generally higher at the SPDC Flow Station site and the LNG Seaport site. There were no significant differences in the concentrations of pollutants under and outside tree canopies at the various sites. The concentrations of pollutants at each site reflected the nature of the activities that were ongoing at the sites and not the differences in growth/morphological attributes of the avenue tree species. There is the need to introduce mitigation measures to check rising concentrations of the gaseous pollutants especially at the SPDC Flow Station site in order to avoid associated health challenges in the future.

\section{References}

Akbari, H. (2002). Shade trees reduce building energy use and $\mathrm{CO}_{2}$ emissions from power plants. Environmental Pollution, 116, 119-126. https://doi.org/10.1016/S0269-7491(01)002 64-0

Behera, S. N., Sharma, M., Aneja, V. P., \& Balasubramanian, R. (2013). Ammonia in the atmosphere: a review on emission sources, atmospheric chemistry and deposition on terrestrial bodies. Environmental Science and Pollution Research, 20(11), 8092-8131. https://doi.org/10.1007/s11356-013-2051-9

Benneth, J. A., \& Hill, A. C. (2013). Absorption of gaseous pollutants by a standardized plant canopy. Journal of the Air pollution Control Association, 23(3), 203-206. https://doi.org/10.1080/00022470.1973.10469767

Chima, U. D., \& Ofodile, E. A. U. (2015). Climate change mitigation and adaptation capabilities of avenue tree species at the University of Port Harcourt, Nigeria. Advances in Applied Science Research, 6(10), 40-49.

Chima, U. D., Akhabue, F. E., \& Gideon, I. K. (2016). Rhizosphere soil properties and growth attributes of four tree species in a four-year arboretum at the University of Port Harcourt, Nigeria. Nigerian Journal of Agriculture, Food and Environment, 12(2), 74-80.

Ede, P. N., \& Edokpa, D. O. (2015). Regional air quality of the Nigeria's Niger Delta. Open Journal of Air Pollution, 4, 7-15. https://doi.org/10.4236/ojap.2015.41002

Efe, S. I., \& Weli, V. E. (2015). Economic impact of climate change in Port Harcourt, Nigeria. Open Journal of Social Sciences, 3, 57-68. https://doi.org/10.4236/jss.2015.33012

EPA. (2010). Methane and Nitrous Oxide Emissions from Natural Sources. U.S. Environmental Protection Agency, Washington, DC, USA. 
Heimlich, J. T., Sydnor, D., Bumgardner M., \& O’ Brien P., (2008). Attitudes of residents towards street trees on four species in Toledo, Ohio, U.G. before removal of ash trees (Fraxinus Spp) from emerald ash borer (Agrilus planipennis) Aboriculture and Urban Forestry, 34(1), 47-53.

Jimoda, L. A. (2012). Effects of particulate matter on human health, the ecosystem, climate and materials: A review. Working and Living Environmental Protection, 9(1), 27-44.

Jones, P. G., \& Thornton, P. K. (2003). The potential impacts of climate change on maize production in Africa and Latin America in 2055. Global Environmental Change, 13(1), 51-59. https://doi.org/10.1016/S0959-3780(02)00090-0

Kumar, S. R, Arumugan, T., Anandakumar, C. R., Balakrishnan, S., \& Rayavet, D. S. (2012). Use of plant species in controlling environmental pollution-a review. Bulletin of Environment, Pharmacology and Life Sciences, 2(2), 52-63.

Intergovernmental Panel on climate change (IPCC) (2001). Impacts, Vulnerability and Adaptation. Contribution of Working Group III to the Third Assessment Report on the intergovernmental panel on climate change. Cambridge UL. Cambridge University Press.

IUCN. (2009). Position paper: Ecosystem-based adaptation (EBA). UNFCCC Climate Change Talks. 28 September - 9 October, Bangkok, Thailand.

Ndubueze-Ogaraku, M.E. and Emeh, (2019). Effect of climate change adaptation on sweet potatoes and three-leaved yam production in Bende Local Government Area of Abia State, Nigeria. African Journal of Agriculture Technology and Environment, 8(1), 26-39.

NIOSH. (1992). Recommendations for occupational safety and health: Compendium of policy documents and statements. Cincinnati, OH : U.S. Department of health and human services, Public Health Service,Centers for Disease Control, National Institute for Occupational Safety and Health, DHHS(NIOSH) Publications No. 92-100.

Roy, R. K., \& Singh, S. (2014). Urban Landscape and amelioration of environment by trees: Method and approaches. International Journal of Latest Research in Science and Technology, 3(3), 98-104.

Scott, K. I., Simpson, J. R., \& McPherson, E. G. (1999). Effects of tree cover on parking lot of microclimate and vehicle emissions. Journal of Aboriculture, 25(3), 129-142.

Scottish Environment Protection Agency (SEPA) (2017). The chemistry of air pollution. https://www.sepa.org.uk/media/120465/mtc_chem_of_air_pollution.pdf. Retrieved $19^{\text {th }}$ November, 2017.

US EPA. (2007). Six common air pollutants: Carbon monoxide. http://www.epa.gov/air/urbanair/co/effrt1.html

U.S. Environmental Protection Agency (2008). Integrated Science Assessment for Oxides of Nitrogen - Health Criteria (Final Report). Washington, DC: National Center for Environmental Assessment. EPA/600/R-08/071.

White, L., \& Edwards, A. (Eds.) (2000). Conservation Research in African rain forests: a technical handbook. Wildlife Conservation Society, New York, 444pp.

Yang, J., McBride, J., Zhou, J., \& Sun, Z. (2005). The urban forest in Beijing and its role in air pollution reduction. Urban Forestry and Urban Greening, 3, 65-78. https://doi.org/10.1016/ 


\section{Macrothink}

j.ufug.2004.09.001

\section{Copyright}

Copyright for this article is retained by the author(s), with first publication rights granted to the journal.

This is an open-access article distributed under the terms and conditions of the Creative Commons Attribution license (http://creativecommons.org/licenses/by/4.0/). 